Geschichte offen, zum zweiten das Projekt des Westens hartnäckig, zum dritten die Gegenwehr ebenso.

Winkler, Chronist und Analytiker der politischen Geschichte zugleich, präsentiert ein profundes Werk, das faktenreich informiert, argumentationsstark urteilt, Zusammenhänge entfaltet und glänzend formuliert, wobei die Steigerung des Wortes „extrem“ (S. 531, S. 594) irritiert. Die „Geschichte des Westens“ ist eine „Geschichte für den Westen“, ohne dass der Autor Apologie betreibt. Was das Einmannunternehmen Winkler in vier Bänden auf fast 5.000 Seiten geleistet hat, ist Herkulesarbeit. Wer jeden Tag fast drei Seiten zu Papier bringt (nur bezogen auf die "Geschichte des Westens“), benötigt langen Atem. Der Respekt des Lesers gehört dem Berliner Historiker, der, im achten Lebensjahrzehnt stehend, innerhalb von sechs Jahren ein solch monumentales Werk vorgelegt hat, selbst wenn Neunmalkluge den einen oder anderen - etwa ideengeschichtlichen - Aspekt missen mögen. Ob diese meisterhafte Geschichte je überboten wird?

Eckhard Jesse

\title{
Bilanz der Föderalismusreform: umfassende Dokumentation mit analytischen Defiziten
}

Schneider, Hans-Peter: Der neue deutsche Bundesstaat. Bericht über die Umsetzung der Föderalismusreform I (Reihe: Föderalismus - Studien, Band 25), Nomos Verlagsgesellschaft, BadenBaden 2013, 829 Seiten, $€ 189$,-.

Am 1. September 2006 traten im Rahmen der ersten Stufe der Föderalismusreform 25 Änderungen beziehungsweise Ergänzungen von Grundgesetzartikeln in Kraft. Die Einschätzung der Resultate ist in Politik und Wissenschaft noch immer umstritten. Zwar sind zu dieser Fragestellung eine Reihe von wissenschaftlichen Publikationen erschienen, was bis dato aber fehlte, waren Studien zur Umsetzung der Verfassungsreform. Der vorliegende Band unternimmt es, dieses Forschungsdesiderat zu schließen.

Auf über 800 Seiten legt der Verfassungsrechtler und Föderalismusforscher Hans-Peter Schneider den Abschlussbericht seines Projekts zur Analyse der umfangreichsten Teilrevision der bundesdeutschen Verfassung vor. Das Forschungsvorhaben wurde von 2008 bis 2012 unter der wissenschaftlichen Mitarbeit von Jutta Kramer und Till Bender am „Deutschen Institut für Föderalismusforschung e.V.“ durchgeführt. Die Forschungsfrage lautete, ob und in welchem Maße die Ziele der Föderalismusreform I erfolgreich umgesetzt wurden und wo weiterer Handlungsbedarf für Änderungen lokalisiert werden könne (S. 24 f.). Methodisch beruht die Analyse auf der Auswertung von über 1.200 schriftlichen Dokumenten. Für das Projekt wurde eigens eine Datenbank aufgebaut. Leider liegt der Publikation keine CD-ROM bei, die einen Zugang zum Datenmaterial ermöglicht.

Der vorgelegte Abschlussbericht ist als Implementations- und Evaluationsstudie angelegt (S. 6). Entsprechend gliedert sich der Band in zwei unterschiedliche Teilbereiche. Der erste sehr umfangreiche - Darstellung und Bestandsaufnahme - hat einen beschreibend dokumentierenden Charakter. Bewusst verzichtet der Autor auf die systematische Einbeziehung der vielgestaltigen wissenschaftlichen Literatur. Den Kern bildet die detaillierte und ausführliche Darstellung der Veränderungen des Grundgesetzes. Der zweite Teil - Auswertung und 
Resümee - will den Erfolg der Reform bewerten. Hans-Peter Schneider misst diesen vor dem Horizont der Ergebnisse des Reformprozesses: Er prüft, ob und inwieweit es gelungen ist, die beschlossenen Verfassungsänderungen im Lichte der Reformziele umzusetzen (S. 730).

Die Untersuchung kommt zu dem Ergebnis, dass sowohl die „Kommission von Bundestag und Bundesrat zur Modernisierung der bundesstaatlichen Ordnung “, die das Reformvorhaben inhaltlich ausgearbeitet hat, als auch die Umsetzung der Reformen durch den Bund und die Länder als ein klarer Erfolg zu bewerten sind (S. 6). Die Föderalismusreform I habe den bundesdeutschen Föderalismus tiefgreifend umgestaltet und seine Funktionsfähigkeit optimiert (S. 749 f.). Der „neue“ deutsche Bundesstaat habe nach der Verfassungsreform ein „Optimum an politischer Handlungs- und Gestaltungsfähigkeit erlangt“ (S. 750).

Die Studie sieht die Ziele der Föderalismusreform I weitgehend umgesetzt (S. 664 ff.): Die Verflechtung von Bund und Ländern ist für eine Vielzahl von Regelungsmaterien beseitigt; die Zahl zustimmungsbedürftiger Gesetze durch den Bundesrat ist erheblich reduziert; Mischfinanzierungen wurden einerseits entflochten und andererseits in einigen Bereichen der Landeszuständigkeit von der Verwaltungs- auf die Gesetzesakzessorietät umgestellt; die Gemeinschaftsaufgaben wurden reformiert und tragen nun zum Abbau von Mischfinanzierungen bei.

Darüber hinaus wird argumentiert, dass sich der Erfolg nicht auf Detailänderungen des Grundgesetzes beschränke. Vielmehr habe die Reform den bundesdeutschen Föderalismus strukturell verändert und verbessert. Das föderative System habe sich vom „kooperativen Föderalismus“ zum „synergetischen Föderalismus“ fortentwickelt (S. 755). Den Typus kennzeichne, dass „die neuen Modelle und Ausprägungen der Zusammenarbeit von Bund und Ländern auf dem Grundsatz der Freiwilligkeit beruhen und durch Verfassung und Bundesgesetz vorgegeben sind“ (S. 755).

Besonders der erste Teil des Bandes leistet einen wichtigen Beitrag zur Einschätzung der Föderalismusreform I. Hier gelingt eine sehr kenntnisreiche, präzise und dichte Darstellung der Veränderungen der einzelnen Normen des Grundgesetzes. Im Blick auf die Umsetzung durch den Bund werden die Neuordnungen der ausschließlichen und konkurrierenden Gesetzgebung (S. 76 - 133) dargelegt: der Wegfall der Rahmengesetzgebung (S. 133 - 139), das Aufgabenübertragungsverbot auf Kommunen (S. 139 - 144), die Veränderungen der Gemeinschaftsaufgaben (S. 144 - 156), der Finanzverfassung (S. 156 - 172), der Hauptstadtregelung (S. 173 f.) und die Verbesserung der Europatauglichkeit (S. 174 - 184). Ein weiterer Abschnitt bildet die rechtlichen Veränderungen durch die Umsetzung der Reform durch die Länder in den Bereichen der ausschließlichen Gesetzgebungskompetenzen (S. 184 - 574) sowie der formellen und materiellen Abweichungsregelungen (S. $574-656$ ) ab. Anschließend werden die verfassungsrechtlichen Modifikationen der Zustimmungsbedürftigkeit in den Sektoren formelle Abweichungsregeln (S. 656 - 660) wie auch neue Zustimmungstatbestände (S. 660 ff.) aufgezeigt und die Entwicklung des Anteils zustimmungsbedürftiger Gesetze im Bundesrat knapp bewertet (S. 662 f.).

Neben diesen Stärken gilt es jedoch auch auf Defizite hinzuweisen. Ein erster wichtiger Kritikpunkt bezieht sich auf die zentrale Aussage der Studie, dass die Föderalismusreform eine Erfolgsgeschichte darstellt. Schneider gelingt es nicht, plausibel darzulegen, dass die Reform der föderalen Struktur der Bundesrepublik Deutschland zu einem Optimum an politischer Handlungs- und Gestaltungsfähigkeit geführt hat. Eine solch weitreichende Einschätzung lässt die Engführung des Bewertungsmaßstabes für die Umsetzung der beschlossenen Reformmaßnahmen nicht zu. Diese beruht lediglich auf immanenten Maßstä- 
ben (S. 733): Umfang der Umsetzung; mögliche Wechselwirkungen; Ausmaß der Zielerreichung; Angemessenheit der Umsetzungsmaßnahmen. Um argumentativ nachvollziehbar das diagnostizierte Optimum an Zielerreichung zu begründen, wäre es erforderlich gewesen, den Bewertungsmaßstab auf wichtige Kontextfaktoren auszudehnen. Diese blendet Schneider jedoch zuvor weitgehend aus: Ursachen, Problemdiagnosen und Anlässe, die zur ersten Stufe der Föderalismusreform führten, und die Analyse der Interessen und Strategien der beteiligten Akteure unter herrschaftssoziologischen Aspekten.

Problematisch sind zweitens die Begriffe „neuer“ deutscher Bundesstaat und „synergetischer Föderalismus“. Beide reklamieren, dass der bundesdeutsche Föderalismus eine tiefgreifende qualitative Transformation erfahren habe. Doch unklar ist, wer Subjekt des Veränderungsprozesses ist. Die Föderalismuskommission hat sich in ihrer Arbeit bekanntlich auf kein gemeinsames Leitbild verständigen können.

Drittens erweist sich die methodische Anlage des Abschlussberichts als Problem. Kritisch anzumerken ist die Fixierung auf schriftliches Quellenmaterial. Für die Analyse der Umsetzung der Verfassungsnormen in die Verwaltungswirklichkeit wurden beteiligte Akteure der Bundes- und Landesverwaltungen nicht systematisch einbezogen. Dies reduziert den Ertrag der Studie. Ein gehaltvolles realistisches Bild des Implementationsgeschehens lässt sich so kaum zeichnen.

Die Einschätzung der Ergebnisse der Föderalismuskommission I bleibt kontrovers. Wünschenswert sind ergänzende Forschungen, die verstärkt interdisziplinär die Probleme des bundesdeutschen Föderalismus untersuchen.

Stephan Bröchler

Gesetzgebungskompetenzen in der Rechtsprechung: Erleichterung der Praxis und Fortschritt der Wissenschaft

Herbst, Tobias: Gesetzgebungskompetenzen im Bundesstaat. Eine Rekonstruktion der Rechtsprechung des Bundesverfassungsgerichts (Schriftenreihe: Jus Publicum), Mohr Siebeck, Tübingen 2014, 412 Seiten, $€ 104$,-.

Eine systematische monographische Behandlung der Gesetzgebungskompetenzen unter dem Grundgesetz war ein Desiderat. Nicht zufällig wird in diesem Zusammenhang auch heute noch die Kommentierung von Christian Pestalozza in der - unvollständig gebliebenen - dritten Auflage des Grundgesetz-Kommentars von von Mangoldt / Klein / Starck herangezogen, die schon umfangmäßig bislang unübertroffen ist. Allein, es fehlte an einer systematischen dogmatischen Erschließung, die - über die verschiedenen einschlägigen Grundgesetzänderungen hinweg - die Rechtsprechung des BVerfG zu einem Sinnganzen zusammengefügt hätte. Diese Arbeit liegt mit der im Jahre 2010 als Habilitationsschrift an der Berliner Humboldt Universität angenommenen Arbeit von Tobias Herbst nun vor. Die in der Reihe Jus Publicum erschienene Schrift wirkt schon äußerlich erfrischend, weil sie nur gut 400 Seiten umfasst und damit zeigt, dass wissenschaftlicher Fortschritt (gerade) auch auf knappem Raum möglich ist. 